\title{
LOCALIZING THE RETINAL HOLE IN THE SURGICAL TREATMENT OF RETINAL DETACHMENT*
}

\author{
BY \\ R. DALGLEISH \\ Department of Ophthalmology, University of Manchester
}

IN the early 1920's Jules Gonin established the principle of sealing retinal holes by producing a thermal reaction in the adjacent choroid. Since that time, the accurate localization of the retinal holes in relation to the outer surface of the sclera has been an essential step in most operations for retinal detachment, and various techniques have been described.

The application of surface diathermy to the sclera, followed by ophthalmoscopic observation of the choroido-retinal reaction, is a time-honoured technique which may be used with shallow detachments; it fails with deep detachments, as no reaction can then be seen with the ophthalmoscope.

Stallard (1958) advocated the focusing of a narrow beam of light from an ophthalmoscope on to the retinal hole; the operator's assistant observes the surface of the sclera and marks the point of transillumination. This technique has obvious limitations, as it is often not possible to position the eye so that both the retinal hole and the corresponding area of sclera can be observed simultaneously.

Strampelli (1935) transilluminated the sclera in the opposite direction by means of a narrow curved tube carrying a transilluminating component and a diathermy terminal. The retina is then observed with an ophthalmoscope and, when the point of maximum illumination is over the tear, a diathermy application is made to mark the sclera.

Schepens, Okamura, and Brockhurst (1957) advocated the use of a flat-tip diathermy electrode on a curved shank. An assistant indents the sclera with the electrode in the approximate region of the hole, while the operator observes the resulting choroidal indentation with an ophthalmoscope. The operator directs the assistant to move the electrode until the indentation coincides with the retinal hole, and a diathermy application is then made to mark the sclera.

In our experience a scleral-indentation technique is the simplest and most reliable way of localizing retinal holes. The choroidal indentation can be observed with equal facility through shallow or deep detachments.

Marking the sclera with a diathermy application, however, has certain disadvantages. Bleeding may make it difficult to keep the point of the electrode dry and in close apposition with the sclera. The scleral reaction is therefore variable and may be difficult to identify. In addition, a diathermy application causes immediate shrinkage of the sclera and necrosis at a later date. Both these factors are undesirable with certain modern techniques for the surgical treatment of retinal detachment. At present, the intra-scleral silicone-rubber implant with encircling silicone-rubber band (Regan, Schepens, Okamura, Brockhurst and McMeel, 1962) is the most commonly used procedure in our Retinal Detachment Clinic. In this operation it is necessary to raise relatively large scleral flaps at the site of the retinal tear (Fig. 1, opposite), and any scleral shrinkage due to the application of surface

\footnotetext{
* Received for publication February 3, 1964.
} 
FIG. 1.-Intra-scleral silicone-rubber implant with encircling silicone-rubber band.

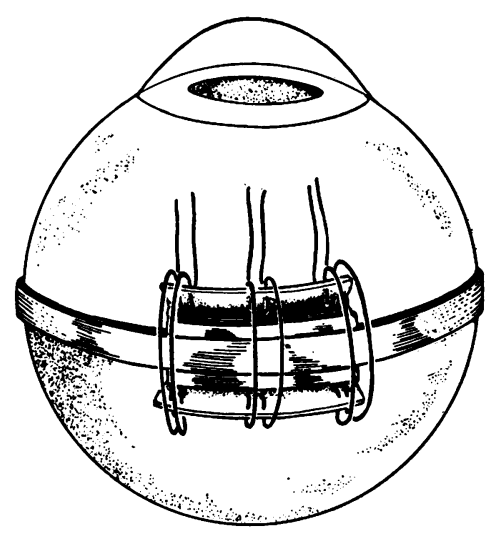

diathermy makes this more difficult. Late necrosis of these flaps or the cutting-out of the sutures which close them is also undesirable, and is another contraindication to surface diathermy to the sclera forming the flaps.

The scleral indentor designed by Urrets-Zavalia (1959) overcomes some of these difficulties. It consists of a straight steel shaft bent at right angles near its extremity. The flat end of this extremity bears a short and not very sharp point at its centre. This point fixes the instrument to the sclera, and thereby enables the globe to be rotated into a suitable position for ophthalmoscopy. We have tried instruments similar to this, and have found this type of fixation to the sclera unsatisfactory. A straight shaft is also a disadvantage, as it is difficult to apply the tip of a straight scleral indentor to areas behind the equator, especially in a region covered by the upper eyelid.

\section{Instrument}

A scleral indentor for localizing retinal holes, which overcomes most of the difficulties already outlined, has been in use in our Retinal Detachment Clinic for more than a year. It is essentially a scleral hook with a curved shank, the plane of the hook being at right angles to the long axis of the handle of the instrument (Fig. 2). The first $2 \mathrm{~mm}$. of shank adjacent to the hook is also at right angles to the long axis of the handle. The next portion of the shank is curved on a radius of $14 \mathrm{~mm}$. The reason for this radius is shown in Fig. 3; it is the radius of the globe $+2 \mathrm{~mm}$. The remainder of the shank forms a compensating curve which brings the hook in line with the long axis of the handle. This promotes rapid orientation of the position of the hook at operation.

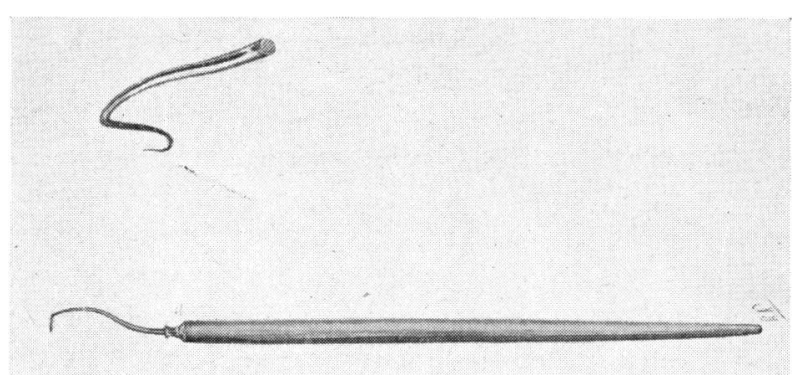

FIG. 2.-The scleral indentor.

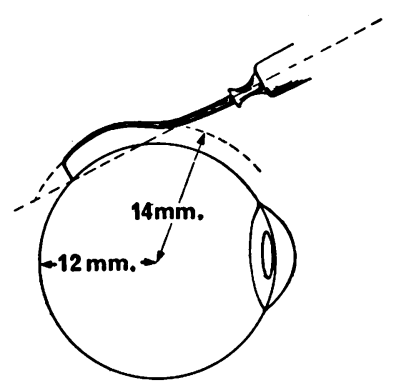

FIG. 3.-The specification of the indentor. 


\section{Technique}

Pre-operatively the usual drawings are made of the retinal detachment showing the position of the hole(s). The pupil is widely dilated.

At operation the sclera is exposed in the appropriate region, and the hook of the scleral indentor is passed through a few superficial scleral lamellae at a site which is judged to correspond with the region of the retinal hole. The instrument is held in the left hand and its long axis is kept in line with the antero-posterior meridian of its site of attachment to the globe; this facilitates orientation of the position of the tip of the indentor. Downward pressure with the instrument rotates the globe into a suitable position for ophthalmoscopy (Fig. 4), which can be direct or indirect in type according to personal preference.

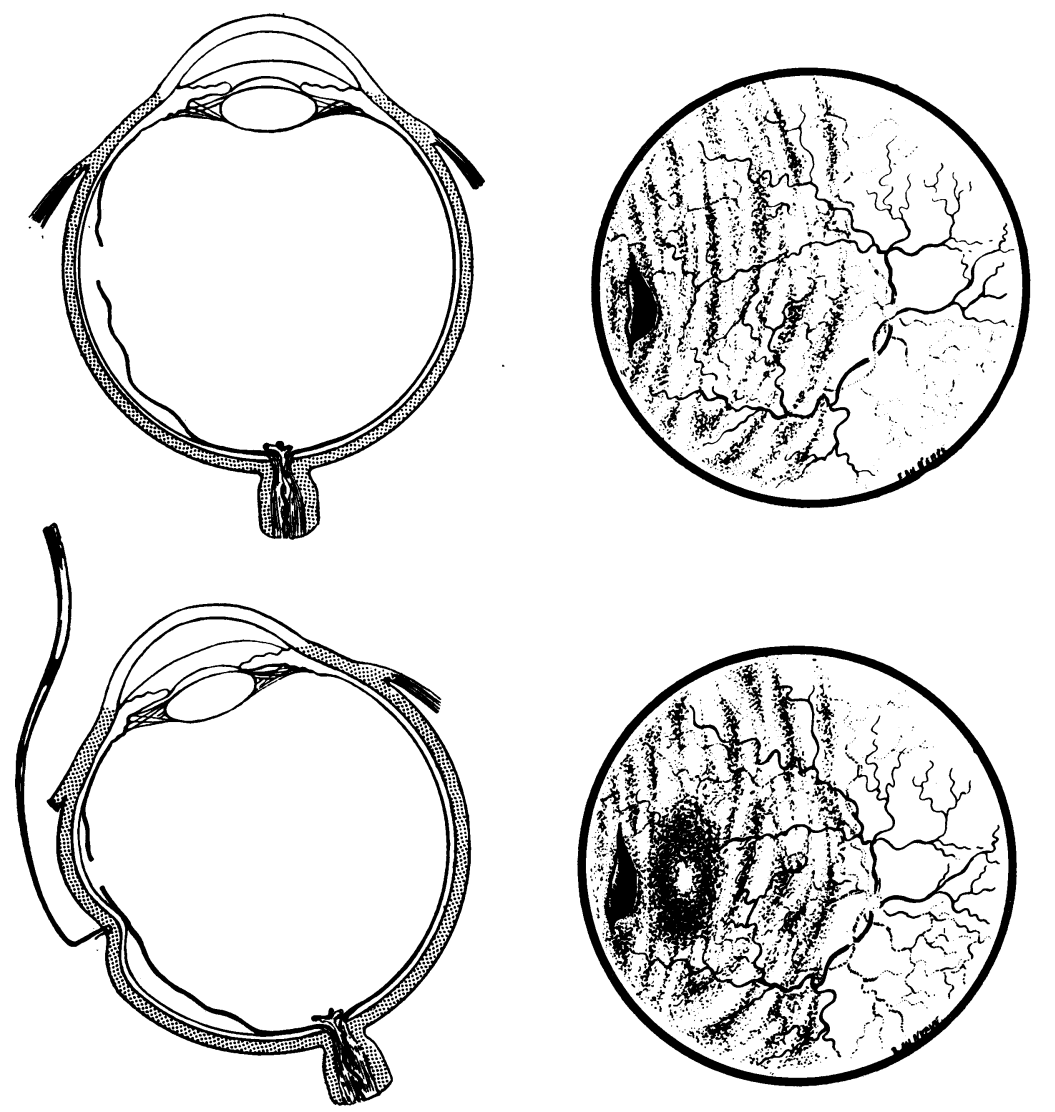

Fig. 4.-The application of the indentor to the sclera and the corresponding appearance of the fundus.

While the retina is under observation, gentle inward pressure is applied with the scleral indentor. It is important to keep the shank of the instrument away from the globe, and to exert light pressure with the tip only. This causes a localized steep indentation, and viewed with the ophthalmoscope this has the appearance of a small dark hillock under the retina, with a somewhat lighter apex (Fig. 4). The position of this indentation is compared with the position of the retinal hole. If the two are 
widely apart, the globe is rotated, and the indentor is re-applied to the sclera at what is judged to be the correct situation. When the indentation corresponds (or nearly corresponds) with the retinal hole, the globe is rotated, the sclera is dried with a gauze swab, and a dot of gentian violet is applied at the appropriate site on the sclera, near to the hook of the instrument, to mark the position of the retinal hole. The indentor is then unhooked.

It must be re-emphasized that it is important to keep the shank of the indentor away from the globe, and that it is only necessary to exert light pressure with the tip of the instrument. Large indentations are undesirable as they cause disorientation by inducing a considerable change in the appearance of a retinal detachment; in addition, large areas of choroido-retinal apposition make the identification of retinal holes difficult.

\section{Summary}

A survey of various techniques for localizing retinal holes in the surgical treatment of retinal detachment is presented. In our experience a scleral indentation technique is the simplest and most reliable method.

A scleral indentor has been designed which overcomes most of the difficulties encountered with the techniques previously described. This instrument has been in use in our Retinal Detachment Clinic for more than a year; during this time all surgically treated retinal holes have been localized with complete accuracy.

Details are presented concerning the construction and use of the indentor.

The instrument is made to the author's specification by C. W. Dixey and Son Ltd., London W.1.

The illustrations were created by Mr. R. L. Neave, Medical Artist, Manchester University, whose skill and co-operation I gratefully acknowledge.

\section{REFERENCES}

Regan, C. D. J., Schepens, C. L., Okamura, I. D., Brockhurst, R. J., and McMeel, J. W. (1962). A.M.A. Arch. Ophthal., 68, 313.

Schepens, C. L., OKamura, I. D., and Brockhurst, R. J. (1957). Ibid., 58, 797.

Stallard, H. B. (1958). "Eye Surgery", 3rd ed., p. 681. Wright, Bristol.

Strampelli, B. (1935). Boll. Ocul., 14, 665.

Urrets-Zavalia, A., Jr. (1959). A.M.A. Arch. Ophthal., 62, 97. 